\title{
一种在无机晶体结构中检索特定子结构的计算机方法
}

\author{
霍卫峰 ${ }^{1}$ 李 $乙^{1, *}$ 卢君然 $^{1}$ 于吉红 ${ }^{1}$ 徐如人 ${ }^{1}$ 李 晶 $^{2}$ \\ ('吉林大学无机合成与制备化学国家重点实验室, 长春 130012; '北京科技大学应用力学系, 北京 100083)
}

\begin{abstract}
摘要：提出了一种针对无机晶体化合物的子结构检索方法. 该方法以 VF2 子图同构算法为基础, 针对无机晶 体化合物的结构特点, 采用了两种策略以提高子结构检索的效率: (1) 引入晶体的对称性信息避免了在等价原 子间进行的大量重复性计算; (2) 采用结构编码预篮选可以有效地减少目标结构的数量. 我们以在无机微孔分 子筛数据库中进行子结构检索为例测试该方法的有效性. 测试结果表明, 该方法可以快速且准确地在分子篮数 据库中检索包含特定子结构的记录. 两种检索策略的引入大大降低了子结构检索的复杂度, 检索速度可提高 3-5个数量级. 该方法通过 Perl 语言实现, 具有较好的可移植性.
\end{abstract}

关键词：无机晶体材料；数据库；子结构检索；VF2算法；分子筛；构筑基元 中图分类号: 0641

\section{A Computational Method for Specified Substructure Search in Inorganic Crystal Structures}

\author{
HUO Wei-Feng ${ }^{1} \quad$ LI Yi ${ }^{1, *} \quad$ LU Jun-Ran ${ }^{1} \quad$ YU Ji-Hong ${ }^{1} \quad$ XU Ru-Ren ${ }^{1} \quad$ LI Jing ${ }^{2}$
}

('State Key Laboratory of Inorganic Synthesis and Preparative Chemistry, Jilin University, Changchun 130012, P. R. China;

${ }^{2}$ Department of Applied Mechanics, University of Science and Technology Beijing, Beijing 100083, P. R. China)

\begin{abstract}
In this paper, a computational method for the substructure search in inorganic crystal structures is proposed. This method is based on the VF2 subgraph isomorphism algorithm. Furthermore, two additional approaches have been introduced into this method to improve the calculation efficiency of VF2: (1) introduction of crystal symmetry information with a view to avoiding redundant calculations among equivalent nodes (atoms); (2) a prescreening encoding treatment to enhance the calculation efficiency by greatly reducing the number of target structures. We tested the efficiency of this method by searching the zeolite crystal structure database from the International Zeolite Association for entries containing specified building units. The test results showed that this method could quickly and correctly retrieve all the entries containing the queried substructure in the zeolite structure database. The introduction of crystal symmetry information and the prescreening encoding treatment greatly reduce the complexity of substructure search. The search speed was significantly enhanced by at least $3-5$ orders of magnitude. This method was developed using Perl programming language, ensuring that this method could be easily applied to various platforms.
\end{abstract}

Key Words: Inorganic crystal structure; Database; Substructure search; VF2 algorithm; Zeolite; Building unit

\section{1 引言}

分子结构检索是利用计算机程序从数据库中
提取分子结构信息的过程, 是化学信息学的核心研

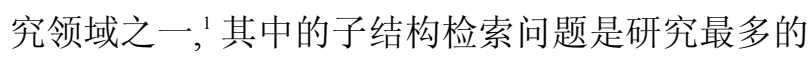

Received: November 17, 2011; Revised: December 27, 2011; Published on Web: January 4, 2012.

"Corresponding author. Email: yili@jlu.edu.cn; Tel: +86-431-85168609.

The project was supported by the National Natural Science Foundation of China (21001049).

国家自然科学基金(21001049)资助项目

(C) Editorial office of Acta Physico-Chimica Sinica 
课题之一. 简单而言, 子结构检索就是利用计算机 方法在分子结构数据库中寻找包含特定子结构的 分子. 目前, 子结构检索主要应用于在有机分子结 构中寻找特定的官能团, 主要有 ParMol、 ${ }^{2} \mathrm{MoFa} 、{ }^{3}$ FFSM、 ${ }^{4}$ gSpan、 ${ }^{5}$ Gaston、 ${ }^{6} \mathrm{SMSD}^{7}$ 等方法. 和有机分 子一样, 无机晶体材料的子结构(构筑基元)对无机 晶体材料的结构、性质以及合成研究至关重要, 8 , 因 此针对无机晶体材料的子结构检索同样具有非常 重要的意义.

然而, 和以独立分子为单位的有机晶体材料不 同, 无机晶体结构往往是三维方向无限延展的, 没 有像有机分子一样的特定独立单元, 数以万计不同 配位状态的原子相互连接形成一个巨大的拓扑网 络结构. 在如此巨大的体系中进行子结构检索是一 个非常复杂的问题; 而当检索范围扩大到由大量复 杂无机结构组成的数据库时, 子结构检索将变得难 以实现. 目前在化学领域中使用最广泛的晶体结构 数据库是剑桥结构数据库 $(\mathrm{CSD}){ }^{10}$ 数据库中包含剑 桥晶体数据中心开发的子结构检索工具 Conquest, ${ }^{11}$ 其原理和有机分子的检索类似, 即把庞大的晶体结 构分割为有限大小的晶体学不对称单元, 进一步把 这些不对称单元当作独立分子进行子结构检索. 这 种检索方法的局限性是显而易见的: 首先, 无机晶 体的不对称单元在理论上是可以任意选取的, 仅凭 不对称单元无法代表整个无机骨架结构; 更为重要 的是, 当待检索的子结构位于一个以上不对称单元 的交界时, 像 Conquest这种只检索不对称单元内部 的方法是无法识别不对称单元之间的关联的. 因此 以晶体学不对称单元或独立分子为基础的子结构 检索方法并不适用于不具备独立单元的无机晶体 结构. 目前, 对无机晶体结构进行子结构检索的唯 一途径是将其骨架拓扑网络扩展到相当大的范围 内(例如 8 个晶胞), 而扩大的拓扑网络同时会使计算 量以指数形式递增, 当检索的对象是包含大量无机 结构的数据库时, 子结构检索将变得难以实现.

由此可见, 无机晶体材料子结构检索的重要性 和理论方法的局限性迫切要求新方法的诞生. 基于 这些问题, 结合无机晶体材料的结构特点, 我们提 出了一种可以在无机晶体结构中有效地进行子结 构检索的方法.

\section{2 方 法}

将无机晶体结构中的中心原子看作节点, 将节
点之间的连接看作边, 无机晶体结构就可以转化成 图论中的图, 子结构检索问题就转化成为图论中的 子图同构问题. 子图同构是非确定多项式时间完全 问题(NPC), 即没有方法可以在合理的时间内(多项 式时间)解决它. ${ }^{12}$ 算法的时间消耗是随节点(中心原 子)数的增加呈指数级增长的, 可以想象, 对于包含 大量原子的无机晶体结构而言, 传统的子图同构算 法是难以实际应用的.

很多研究尝试将子图同构算法的复杂程度降 至可以实际应用的程度, 主要的算法包括 Ullmann 算法、 ${ }^{13} \mathrm{SD}$ 算法、 ${ }^{14}$ Nauty 算法、 ${ }^{15} \mathrm{VF}$ 算法 ${ }^{16}$ 和 VF2 算 法 ${ }^{17}$ 等. 其中, $V F 2$ 算法是在 $V F$ 算法的基础上发展 起来的, 和 VF 算法相比, VF2 算法成功地将空间复 杂度从 $O\left(N^{2}\right)$ 降到了 $O(N)$, 其中 $N$ 为图中的节点数. 特别是在规则图(每个节点都具有相同数目邻接节 点的图)和大图(节点数大于 600 的图)的同构计算 中, VF2 算法具有比其他算法更高的性能. ${ }^{1,18-21}$ 因为 无机晶体的拓扑网络结构绝大多数属于规则图和 大图, 所以我们采用 VF2 子图同构算法作为子结构 检索方法的基础.

VF2 算法是一种深度优先的回溯算法, 算法的 高效性得益于采用了状态空间表示(SSR)的方法和 减少搜索空间的一些匹配规则. ${ }^{16}$

图 1 为检索图 $(Q)$ 和目标图 $(T)$ 示意图. 如图 1 所 示, 假设要查询的图 $Q$ 中有 $N_{1}$ 个节点, 目标图 $T$ 中有 $N_{2}$ 个节点, $N_{1} \leq N_{2} . M$ 是 $Q$ 和 $T$ 之间的映射, 包含点对 $(q, t)$, 其中, $q \in Q, t \in T, M \subset N_{1} \times N_{2}$. 状态空间 $S$ 是 $M$ 的一个子集, 用于存储图 $Q$ 和图 $T$ 的局部匹配. 点对 $\left(q_{i}, t_{i}\right)$ 是否可以加入 $S$, 取决于其是否满足匹配规则. 在这里, 我们规定点对 $\left(q_{i}, t_{i}\right)$ 加入 $S$ 的规则是: (1) $q_{i}$ 与 $t_{i}$ 的类型要一致; (2) 对于 $S$ 中任一已有的点对 $\left(q_{i-1}, t_{i-1}\right)$, 如果 $q_{i}$ 与 $q_{i-1}$ 相连, 则 $t_{i}$ 和 $t_{i-1}$ 必须相连; (3) $t_{i}$ 的邻接节点数要小于或等于 $q_{i}$ 的邻接节点数.

状态空间 $S$ 的初值 $S_{0}$ 为空. 从查询图 $Q$ 中选择 任意一个未访问过的节点 $q_{1}$, 在目标图 $T$ 中寻找当 前这一轮未被访问过的且满足匹配规则的对应节
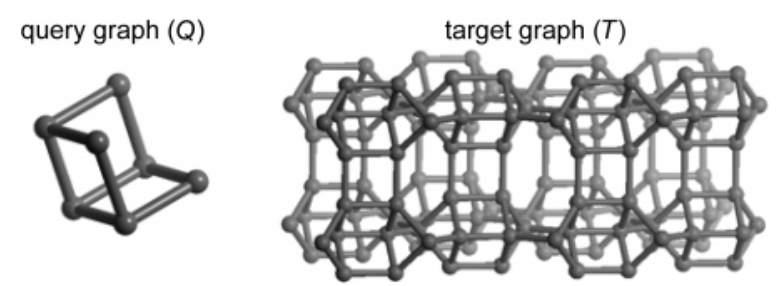

图 1 检索图 $(Q)$ 和目标图(T)示意图

Fig.1 Diagrams of query graph $(Q)$ and target graph $(T)$ 
点. 如果 $Q$ 中的第一个节点 $q_{1}$ 没有在 $T$ 中找到对应 的节点, 则 $Q$ 不是 $T$ 的子图; 如果能找到这样的节点 $t_{1}$, 就将点对 $\left(q_{1}, t_{1}\right)$ 放入状态空间 $S$ 中, 然后从节点 $q_{1}$ 在 $Q$ 中的后继节点集(与节点 $q$ 相连的节点集合)中 选择一个节点 $q_{2}$, 再从节点 $t_{1}$ 在 $T$ 中的后继节点集 (与节点 $t_{1}$ 相连的节点集合)中寻找满足匹配规则的 对应节点. 如果成功找到对应节点 $t_{2}$, 则将点对 $\left(q_{2}\right.$, $\left.t_{2}\right)$ 放入到状态空间 $S$ 中; 如果找不到就回溯到上一 轮, 开始新的查找. 当状态空间 $S$ 中有 $N_{1}$ 个点对时, 表明图 $Q$ 是图 $T$ 的子图, 输出结果.

因为无机晶体结构中包含的节点数是非常庞 大的, 直接采用 VF2 算法对无机晶体结构进行检索 仍然是不现实的. 在此基础上, 根据无机晶体材料 的结构特点, 我们在 VF2 算法的基础上做了两个重 要的改进以提高检索效率.

(1) 利用晶体结构的对称性. 在进行匹配前, 分 别将待检子结构片段 (查询图 $Q$ ) 和目标晶体结构片 段(目标图 T)中的晶体学独立或不对称的原子(节 点)移至相应图的顶部. 在进行子图匹配时, 检验 $Q$ 中是否有与 $T$ 中晶体学独立节点相匹配的独立节 点, 如果没有找到满足匹配规则的节点, 就可以认 为待检结构不是目标结构的子结构, 而并不需要遍 历待查询的子结构及目标结构中的所有节点, 从而 大大提高计算的效率.

(2) 采用编码预䇥选方法. 由于子图匹配算法的 计算量非常大, 因此如果在进行子图匹配之前能够 预篮选掉大量确定无效的数据而仅保留少量有可 能符合检索条件的数据, 将极大提高子结构检索的 效率. 为此, 我们设计了一个具有特定位数的二进 制编码, 编码中的每一位对应一种特定的构筑基 元. 以无机微孔分子篮晶体结构为例来说, 我们采 用了一个19位的二进制编码, 编码中的每一位代表 了 19 种在分子篮结构中最常见的构筑基元 ${ }^{22}$ (表 1 ), 编码中每一位的值 1 或 0 代表该结构中含有或不含 有指定的构筑基元. 在进行子图匹配计算之前, 我 们先通过计算机程序把待查询的子结构和目标结 构分别转换成编码(如图 2 所示), 然后对比编码中相 应位上的值, 如果待查询子结构编码中的某一位显 示含有某个特定的构筑基元而目标结构编码显示 不含有, 说明待查询子结构中包含目标结构中所不 包含的构筑基元, 因此可以直接判定目标结构不包 含待查询的子结构; 如果待查询的子结构中所有的 构筑基元在目标结构编码中都显示含有, 则说明目
表 119 个分子篮结构编码中各位所代表的构筑基元

Table 1 Prescreening code for 19 zeolite structure building units

Bit of code Building unit ${ }^{22}$ Bit of code

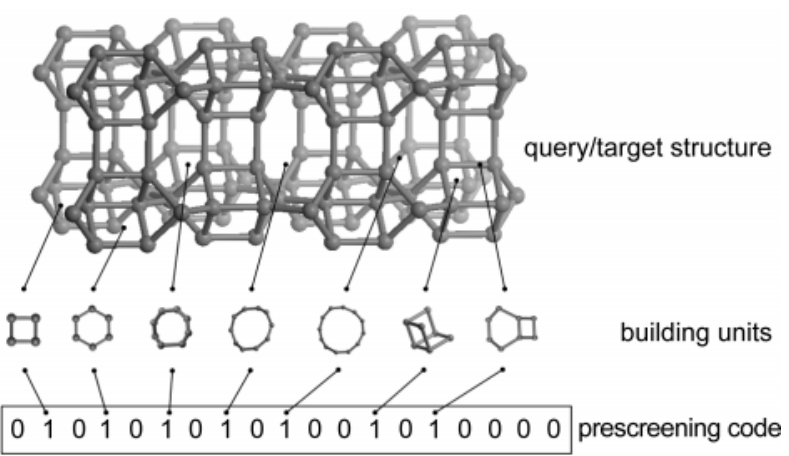

图 2 结构编码

Fig.2 Structure encoding

标结构有可能包含待查询的子结构, 需要进一步通 过子图匹配计算以确定两者是否具有包含关系. 对 于仅包含少量目标结构的数据库而言, 编码预篮选 的优越性并不明显. 随着数据库中目标结构数量的 增加, 编码预笁选的优越性将迅速显现出来. 需要 强调的是, 目标结构的编码转换是在子结构检索之 前完成并保存的, 并不占用子图匹配计算的时间.

\section{3 结果与讨论}

我们以在分子篮晶体结构中检索特定的子结 构为例测试这种方法的效率. 数据源采用了国际分 子篮协会(IZA)官方网站提供的 197 种分子篮结构 
表 2 采用不同策略进行子结构检索所需时间的比较

Table 2 Time needed for substructure search by different approaches

\begin{tabular}{|c|c|c|c|c|c|c|c|}
\hline \multirow[b]{2}{*}{ Building unit } & \multirow[b]{2}{*}{ IZA name } & \multirow[b]{2}{*}{$\begin{array}{l}\text { Number of } \\
\text { nodes }\end{array}$} & \multirow[b]{2}{*}{$\begin{array}{c}\text { Occurrence } \\
\text { frequency in IZA } \\
\text { database }\end{array}$} & \multicolumn{4}{|c|}{ CPU time/s } \\
\hline & & & & $\begin{array}{l}\text { Basic VF2 with no } \\
\text { further treatment }\end{array}$ & $\begin{array}{l}\text { VF2 plus } \\
\text { symmetry } \\
\text { information }\end{array}$ & $\begin{array}{l}\text { VF2 plus } \\
\text { prescreening } \\
\text { encoding } \\
\end{array}$ & $\begin{array}{l}\text { VF2 plus symmetry } \\
\text { and encoding }\end{array}$ \\
\hline & lov & 5 & 8 & 141.0 & 2.0 & 0.2 & 0.1 \\
\hline & nat & 6 & 3 & 5214.8 & 30.1 & 19.4 & 0.5 \\
\hline & $v s v$ & 6 & 10 & 2166.6 & 11.0 & 9.0 & 0.3 \\
\hline & bre & 10 & 9 & 8873.7 & 66.7 & 7.5 & 2.0 \\
\hline & bea & 10 & 5 & 2748.5 & 23.0 & 1272.7 & 17.3 \\
\hline & & 7 & 73 & 10671.8 & 34.0 & 54.3 & 3.3 \\
\hline & & 10 & 146 & 3585.8 & 24.9 & 3571.3 & 23.5 \\
\hline & & 28 & 1 & 10001.7 & 60.4 & 4690.6 & 8.0 \\
\hline
\end{tabular}

的 cif文件. ${ }^{22}$ 从每个结构的 cif文件出发, 计算了 8 个 晶胞内容所对应的邻接矩阵并合并在一起形成结 构数据库. 选取了一些已知的分子耖构筑基元作为 待查询的子结构片段, 在由 197 种分子篎晶体结构 所组成的数据库中进行检索, 并将检索结果与分子 笁协会官方网站提供的手工检索结果相对比, 以此 检验我们这种检索方法的准确性. 在此基础上, 我 们又任意定义了一些新的构筑基元, 进一步考察这 种结构检索方法在不同条件下的效率. 测试的硬件 环境是 Intel Core2 Duo T9600 2.8 GHz, 4 GB DDR2 $800 \mathrm{MHz}$, 操作系统为 Windows 7 Ultimate SP1 32 位, 程序开发环境为 ActivePerl 5.12.4.

选取的已知分子篮构筑基元包括lov、nat、vsv、 bre、bea 等, 22 部分测试结果列在表 2 中. 国际分子篮 协会经过人工分析, 在其官方数据库中给出了这些 常见的构筑基元在 197 个分子笚结构中出现的情 况. 经过对比发现, 采用我们的子结构检索方法得 到的结果与国际分子篮协会人工统计的结果完全 一致. 说明我们的子结构检索方法在准确性上是可 靠的.

此外, 表 2 还列出了各种检索策略所需的 CPU 时间, 包括单纯的 VF2 检索、引入对称性的 VF2 检 索、采用编码预篮选的 VF2 检索以及同时引入对称 性和编码预篎选的 VF2 检索. 尽管这四种策略所得 到的检索结果完全一致, 但检索效率却相差较大. 单纯的 VF2 算法耗时非常长, 在由 197 个结构组成
的数据库中进行子结构检索一般需要 $1 \mathrm{~h}$ 以上的 CPU 时间. 而在引入了对称性信息之后, 检索消耗 的时间会降低 2-3 个数量级. 从理论上说, 对称性的 引入可以大大降低 VF2 算法所需遍历的节点个数, 因此 VF2 算法的效率会得到指数级的提升. 此外, 编码预篎选能够在子图同构计算之前大幅度缩小 目标结构的数量, 同样也可以大幅度提高结构检索 的效率. 需要说明的是, 待检的结构片段越特殊、越 少见, 编码预篎选提升的效率就越高, 这个特性恰 恰可以满足实际需求. 因为在实际应用中, 人们往 往对新颖少见的子结构更关心, 更需要在数据库中 挖掘其结构特殊性. 我们提出的采用编码预䇻选的 子图同构算法恰恰可以有效地解决这类问题. 引入 晶体结构对称性和编码预篮选这两套方案分别基 于不同的原理, 因此将这两种方法同时结合在一 起, 可以将子结构检索的效率进一步提升. 从表 2 的 最终结果可以看出, 同时采用这两种改进方法的检 索策略是效率最高的, 对于简单的结构通常可以在 $1 \mathrm{~s}$ 之内完成检索; 对于相对复杂的结构, 检索时间 会略有增加, 但仍在实际可接受的范围内.

\section{4 结 论}

提出了一种针对无机晶体材料的子结构检索 方法. 该方法基于子图同构的 VF2 算法, 利用晶体 结构的对称性和编码预篮选策略降低结构检索的 复杂度, 可以在无机晶体数据库中快速准确地检索 
包含特定子结构的记录, 为无机晶体材料的结构分 析提供了重要的研究工具. 本文以分子篮结构为例 展示了这种结构检索方法的效率. 除此之外, 该方 法同样可以用于其他无机晶体结构的检索, 包括在 假想分子篮结构数据库、 ${ }^{23}$ 多配位态的开放骨架结 构数据库、 ${ }^{24}$ 甚至致密的矿物晶体结构数据库中进 行的子结构检索. 采用 Perl 语言实现了这种检索方 法, 其高度的可移植性使得这种检索方法可以在绝 大多数计算机平台上得以实现.

\section{References}

(1) Rijnbeek, M.; Steinbeck, C. J. Cheminf. 2009, 1, 17.

(2) Worlein, M. Extension and Parallelization of a Graph-Mining-Algorithm. Ph.D. Dissertation, Friedrich-Alexander-Universität Erlangen-Nürnberg, Germany, 2006.

(3) Meinl, T.; Borgelt, C.; Berthold, M. R. Discriminative Closed Fragment Mining and Perfect Extensions in MoFa. In Frontiers in Artificial Intelligence and Applications; Onaindia, E., Staab, S. Eds.; IOS Press: Amsterdam, 2004; Vol. 109, pp 3-14.

(4) Huan, J.; Wang, W.; Prins, J. Efficient Mining of Frequent Subgraphs in the Presence of Isomorphism. In Proceedings of the 3rd IEEE International Conference on Data Mining, Melbourne, FL, USA, Nov 19-22, 2003; Wu, X. D., Tuzhilin, A., Shavlik, J. Eds.; IEEE Computer Soc.: Los Alamitos, CA, 2003.

(5) Yan, X. F.; Han, J. W. gSpan: Graph-Based Substructure Pattern Mining. In Proceedings of 2002 IEEE International Conference on Data Mining, Maebashi City, Japan, Dec 9-12, 2002; Kumark, V. Ed.; IEEE Computer Soc.: Los Alamitos, CA, 2002.

(6) Nijssen, S.; Kok, J. N. Electronic Notes in Theoretical Computer Science 2005, 127 (1), 77.

(7) Rahman, S. A.; Bashton, M.; Holliday, G. L.; Schrader, R.; Thornton, J. M. J. Cheminf. 2009, 1, 12.

(8) Sastre, G.; Vidal-Moya, J. A.; Blasco, T.; Rius, J.; Jordá, J. L.; Navarro, M. T.; Rey, F.; Corma, A. Angew. Chem. Int. Edit. 2002, 41,4722 .

(9) Corma, A.; Rey, F.; Valencia, S.; Jordá, J. L.; Rius, J. Nature Mater. 2003, 2, 493.

(10) Cambridge Structure Database. http://www.ccdc.cam.ac.uk/ products/csd/ (accessed Aug 30, 2011).

(11) Bruno, I. J.; Cole, J. C.; Edgington, P. R.; Kessler, M. K.; MacRae, C. F.; McCabe, P.; Pearson, J.; Taylor, R. Acta Crystallogr. B: Struct. Sci. 2002, 58, 389.

(12) Cook, S. A. The Complexity of Theorem-Proving Procedures. In Proceedings of the Third Annual ACM Symposium on the Theory of Computing, Ohio, USA, May 3-5, 1971; Harrison, M. A., Banerji, R. B., Ullman, J. D. Eds.; ACM: New York, 1971.

(13) Ullmann, J. R. J. Assoc. Comput. Mach. 1976, $23,31$.

(14) Schmidt, D. C.; Druffel, L. E. J. Assoc. Comput. Mach. 1976, 23, 433.

(15) McKay, B. D. Congressus Numerantium 1981, 30, 45.

(16) Cordella, L. P.; Foggia, P.; Sansone, C.; Vento, M. Performance Evaluation of the VF Graph Matching Algorithm. In Proceedings of the 10th International Conference on Image Analysis and Processing, Venice, Italy, Sept 27-29, 1999; Roberto, G., Cantoni, V., Levialdi, S. Eds.; IEEE Computer Society Press: Los Alamitos, 1999.

(17) Cordella, L. P.; Foggia, P.; Sansone, C.; Vento, M. IEEE Transactions on Pattern Analysis and Machine Intelligence 2004, $26(10), 1367$.

(18) Su, Z. Q.; Liao, C. Z.; Xie, A. H.; Lu, X. P.; Shi, L. M. Computers and Applied Chemistry 2003, 20 (5), 556. [苏振 强, 廖晨钟, 谢爱华, 鲁先平, 石乐明. 计算机与应用化学, 2003, 20 (5), 556.]

(19) Li, X.; Song, T. T.; He, X. F. Computers and Applied Chemistry 2007, 24 (11), 1551. [李 欣, 宋婷婷, 何险峰. 计算机与应用 化学, 2007, 24 (11), 1551.]

(20) Song, T. T.; He, X. F.; Wen, H. Computers and Applied Chemistry 2008, 25 (9), 1152. [宋婷婷, 何险峰, 温 浩. 计算 机与应用化学, 2008, 25 (9), 1152.]

(21) Feng, H. J.; Wang, Y.; Zhou, J. L.; Haji, A. Computer Applications and Software 2010, 27 (10), 117. [冯红君， 汪 渏, 周俊林, 阿吉艾克拜尔・艾萨. 计算机与应用软件, 2010, 27 (10), 117.]

(22) The Database of Zeolite Structures. http://www.iza-structure.org (accessed Aug 30, 2011).

(23) Li, Y.; Yu, J. H.; Xu, R. R. Hypothetical Zeolite Database. http:// mezeopor.jlu.edu.cn/hypo/ (accessed Aug 30, 2011).

(24) Li, Y.; Yu, J. H.; Xu, R. R. AlPO Database. http://mezeopor.jlu. edu.cn/alpo/ (accessed Aug 30, 2011). 